\title{
Insights into the hepatocellular carcinoma patient journey: results of the first global quality of life survey
}

\author{
Jennifer Gill*,1, Andrei Baiceanu², Paul J Clark³, Andrew Langford4, Julianah Latiff ${ }^{5}$, \\ Pei-Ming Yang ${ }^{6}$, Eric M Yoshida ${ }^{7}$ \& Panos Kanavos ${ }^{1}$ \\ ${ }^{1}$ London School of Economics \& Political Science, Houghton St, London WC2A 2AE, UK \\ 2European Liver Patients' Association, Rue de la Loi 235/27 1040 Brussels, Belgium \\ ${ }^{3}$ School of Medicine, University of Queensland, 288 Herston Road, Herston, QLD, 4006, Australia \\ ${ }^{4}$ British Liver Trust, 6 Dean Park Crescent, Bournemouth BH1 1HL, UK \\ ${ }^{5}$ Singapore General Hospital, Outram Road, 169608, Singapore \\ ${ }^{6}$ Taiwan Good Liver Foundation, No.100, Tzyou 1st Road Kaohsiung 807, Taiwan \\ ${ }^{7}$ Canadian Liver Foundation, Suite 801, 3100 Steeles Avenue East Markham, ON L3R 8T3 Canada \\ *Author for correspondence: Tel.: +44 0203486 2609; Fax: +44 0207955 7980; j.gill7@|se.ac.uk
}

\begin{abstract}
Aim: To better understand the hepatocellular carcinoma (HCC) patient journey, we conducted a patient survey across 13 countries. Methods: The survey included closed- and open-ended questions developed using an iterative process to gather information on demographics, diagnosis and treatment. Patients selfselected or were directed to the online survey by their doctor. Results: A total of 256 patients completed the survey. More than two-thirds (68\%) felt they did not receive enough information about HCC at diagnosis. Treatments included oral anticancer therapy, transarterial chemoembolization (TACE), and selective internal radiation therapy (SIRT). A total of $81 \%$ receiving sorafenib, $45 \%$ receiving SIRT and $32 \%$ receiving TACE reported impaired quality-of-life (QoL). A total of 42,19 and $0 \%$ of patients using sorafenib rated their current QoL as 'poor', 'good' and 'excellent', respectively; compared with SIRT (22, 33 and 6\%) or TACE (11, 37 and 13\%). Conclusion: Most patients with HCC require additional accessible information. People with incurable HCC require treatments that preserve QoL.
\end{abstract}

First draft submitted: 19 December 2017; Accepted for publication: 9 February 2018; Published online: 15 March 2018

Keywords: carcinoma $\bullet$ global survey $\bullet$ hepatocellular $\bullet$ patient journey $\bullet$ patient survey $\bullet$ quality of life $\bullet$ SIRT • sorafenib • TACE • treatment effects

Hepatocellular carcinoma (HCC) is the most common primary liver malignancy, the sixth most prevalent cancer globally and the second most frequent cause of cancer-related death worldwide [1-4]. Despite this, the literature contains limited information on the journey of those diagnosed with HCC due to a lack of patient-targeted surveys.

The distribution of HCC varies by geographical location. The incidence in North and South America is relatively low $(<5 / 100,000)$ but this increases to $>20 / 100,000$ in sub-Saharan Africa and East Asia [4,5]. Such discrepancies are likely due to the multifactorial etiology of HCC, including the prevalence of cirrhosis and chronic hepatitis virus infection [3,6].

Prognosis and treatment success depends on accurate staging of HCC and the degree of liver dysfunction. The Barcelona Clinic Liver Cancer Classification (BCLC) system integrates liver cancer stage and disease severity to determine treatment [7], with median survival ranging from $>3$ years for untreated patients with very early HCC (Barcelona Clinic liver cancer stage 0 ) to $<4$ months for those with very advanced disease (BCLC stage D) [8].

For those with very early or early-stage disease (BCLC stage 0 or A), ablation, resection or transplant can often be curative [3,9]. Patients with intermediate-stage disease (BCLC stage B) are generally offered transarterial chemoembolization (TACE) [10]. Intermediate-stage patients unsuitable for TACE may also be offered systemic oral anticancer therapy with sorafenib (Nexavar, Bayer AG, Germany and Onyx Pharmaceuticals Inc, USA), a tyrosine kinase inhibitor. Sorafenib is also the standard of care for patients with advanced disease (BCLC stage C), but 
it has a high frequency of adverse events [11,12]. Those with terminal disease (BCLC stage D) are given palliative treatment [9].

Liver-directed selective internal radiation therapy (SIRT) using ${ }^{90}$ yttrium microspheres is a loco-regional treatment for selected patients with intermediate-to-advanced HCC. Preliminary results from two large-scale trials have recently suggested that SIRT has a similar impact on overall survival as sorafenib but leads to fewer and less severe adverse events [13,14].

Besides therapeutic interventions, information and support plays a vital role in helping patients with HCC maintain their quality of life (QoL). Multiple support groups exist for people with general liver conditions that offer information on HCC (e.g., British Liver Trust, German Liver Aid, Australian Liver Foundation, and American Liver Foundation). However, there are few organizations that focus solely on patients with HCC such as Blue Faery: The Adrienne Wilson Liver Cancer Association (USA). Patients with HCC may be denied valuable support and information which could improve their patient journey.

The aim of this global survey, the first of its kind, was to better understand the patient journey and to compare the effects of later-stage HCC treatments on QoL.

\section{Patients \& methods}

\section{Survey goals \& development}

The objective of the survey was to obtain patient specific-information about personal characteristics (prediagnosis condition, length of time since HCC diagnosis), care processes (i.e., types of therapy received) and outcomes (i.e., QoL) that can be reliably obtained from patients themselves. To ensure validity, the survey was developed via an iterative process between seven medical charities (American Liver Foundation, Blue Faery, British Liver Trust, Canadian Liver Foundation, European Liver Patients Association, Romanian Association of Patients with Liver Diseases, and Taiwan Good Liver Foundation) and >15 medical centers across 13 countries (Argentina, Australia, Brazil, Canada, France, Italy, Germany, Romania, Singapore, Spain, Taiwan, UK and USA). The tool contained 32 open-ended and closed-ended questions covering patient demographics, diagnosis and treatment. Approval of the tool was sought from the scientific advisory boards of the medical centers and patient organizations involved in the project. In Canada, Australia and Spain approval from an independent ethics committee was sought. Survey design followed standard research methodology processes [15].

With the assistance of Strategic Sight (UK), the survey instrument was hosted online at hcc-voices.com from November 2016 to April 2017, where it was made available in eight languages (Chinese, English, French, German, Italian, Portuguese, Romanian and Spanish). Paper copies of the survey were also available. The complete survey is available online [16]. All survey responses were confidential.

\section{Patient recruitment}

The patient charities involved in the project partnership encouraged patients to participate. Patients were either able to self-select for completion after seeing survey-related materials or were asked by the nurses in their specialist doctor's office to complete the survey. Patients over 18 years with a diagnosis of HCC were included.

\section{Data analysis}

The surveys were completed anonymously. For online surveys, results were automatically captured by the online platform. Hardcopy surveys were collated and transcribed into an online form. All survey results were translated into English using Cicero Translations. They were then back-translated to check for accuracy.

On completion, data were exported to Excel for analysis purposes. An online, freely available tool was used to build the word clouds [17].

\section{Results}

Patient characteristics

A total of 256 patients completed the survey across 13 countries. Out of these, 131 were from Europe (France $\mathrm{n}=1$, Germany $\mathrm{n}=23$, Italy $\mathrm{n}=62$, Romania $\mathrm{n}=7$, Spain $\mathrm{n}=6$ and the $\mathrm{UK} \mathrm{n}=32$ ), 64 were from The Americas (Canada $\mathrm{n}=8$, USA $\mathrm{n}=52$, Brazil $\mathrm{n}=4$ ), and 61 were from Asia-Pacific (Australia $\mathrm{n}=1$, Singapore $\mathrm{n}=18$, Taiwan $\mathrm{n}=42$ ). Respondent characteristics are shown in Table 1. The median age of respondents was 64 (range: 23-89) years, with European respondents tending to be slightly older. 


\begin{tabular}{|c|c|c|c|c|c|}
\hline Characteristics & Variables & Global $(n=256)$ & Europe $(n=131)$ & $\begin{array}{l}\text { The Americas } \\
(n=64)\end{array}$ & Asia-Pacific $(n=61)$ \\
\hline \multirow{7}{*}{$\begin{array}{l}\text { Age (years) } \\
\mathrm{N}(\%)\end{array}$} & $20-29$ & $2(0.7)$ & $0(0)$ & $1(1.6)$ & $1(1.6)$ \\
\hline & $30-39$ & $9(4)$ & $5(3.8)$ & $1(1.6)$ & $3(5)$ \\
\hline & $40-49$ & $15(6)$ & $7(5.3)$ & $2(3.1)$ & $6(10)$ \\
\hline & $50-59$ & $62(24)$ & $34(26)$ & $17(26.6)$ & $11(18)$ \\
\hline & $60-69$ & $91(36)$ & $34(26)$ & $34(53.1)$ & $23(38)$ \\
\hline & $70-79$ & $59(23)$ & $7(28)$ & $8(12.5)$ & $14(23)$ \\
\hline & $80-89$ & $18(7)$ & $14(10)$ & $1(1.6)$ & $3(5)$ \\
\hline \multirow[t]{2}{*}{ Sex N (\%) } & Male & $178(70)$ & $88(67)$ & $47(73.4)$ & $43(70)$ \\
\hline & Female & $78(30)$ & $43(33)$ & $17(26.6)$ & $18(30)$ \\
\hline \multirow{6}{*}{$\begin{array}{l}\text { Diagnosing doctor } \\
\mathrm{N}(\%)\end{array}$} & GP & $39(15)$ & $20(15)$ & $9(14)$ & $10(16)$ \\
\hline & Oncologist & $18(7)$ & $11(8)$ & $5(7.8)$ & $2(3)$ \\
\hline & Hepatologist & $109(43)$ & $46(35)$ & $25(39)$ & $38(62)$ \\
\hline & Gastroenterologist & $64(25)$ & $37(28)$ & $20(31.3)$ & $7(11)$ \\
\hline & ER physician & $7(3)$ & $6(5)$ & $1(1.6)$ & $0(0)$ \\
\hline & Other & $25(6)$ & $7(5)$ & $4(6.3)$ & $4(9)$ \\
\hline \multirow{2}{*}{$\begin{array}{l}\text { Received enough information at } \\
\text { diagnosis? N (\%) }\end{array}$} & Yes & $81(32)$ & $33(25)$ & $24(37.5)$ & $24(39)$ \\
\hline & No & $175(68)$ & $98(75)$ & $40(62.5)$ & $37(61)$ \\
\hline \multirow[t]{5}{*}{ Precondition N (\%) } & Chronic HBV & $60(23)$ & $17(13)$ & $3(4.7)$ & $40(66)$ \\
\hline & Chronic HCV & $97(38)$ & 49 (37) & $38(59.4)$ & $10(16)$ \\
\hline & NAFLD & $24(9)$ & $12(9)$ & $9(14.1)$ & $3(5)$ \\
\hline & Other & $45(18)$ & $35(27)$ & $7(10.9)$ & $3(5)$ \\
\hline & No previous condition & $30(12)$ & $18(14)$ & $7(10.9)$ & $5(8)$ \\
\hline \multirow[t]{4}{*}{ Years since diagnosis $\mathrm{N}(\%)$} & $<1$ & $84(33)$ & $48(37)$ & $19(29.7)$ & $17(28)$ \\
\hline & $1-3$ & $88(34)$ & $51(39)$ & $21(32.8)$ & $16(26)$ \\
\hline & $4-6$ & $42(16)$ & $18(14)$ & $11(17.2)$ & $13(21)$ \\
\hline & $>6$ & $42(16)$ & $14(11)$ & $13(20.3)$ & $15(25)$ \\
\hline \multirow[t]{11}{*}{ Treatments received $\mathrm{N}(\%)$} & Transplant & $52(21)$ & $20(15)$ & $25(39)$ & $8(13)$ \\
\hline & Surgery & $75(29)$ & $28(21)$ & $17(26.6)$ & $30(49)$ \\
\hline & Ablation & $85(33)$ & $39(30)$ & $16(25)$ & $30(49)$ \\
\hline & External radiotherapy & $11(4)$ & $6(5)$ & $3(4.7)$ & $2(3)$ \\
\hline & SIRT & $33(13)$ & $12(9)$ & $14(21.9)$ & $8(13)$ \\
\hline & TACE & $111(43)$ & $59(45)$ & $20(31.3)$ & $31(51)$ \\
\hline & Sorafenib & $46(18)$ & $26(20)$ & $12(18.8)$ & $8(13)$ \\
\hline & iv. chemotherapy & $10(4)$ & 7 (5) & $2(3.1)$ & $1(2)$ \\
\hline & Alternative medicine & $15(6)$ & $8(6)$ & $4(6.3)$ & $3(5)$ \\
\hline & No treatment offered & $10(4)$ & $9(7)$ & $1(1.6)$ & $0(0)$ \\
\hline & Treatment declined & $5(2)$ & $3(2)$ & $0(0)$ & $2(3)$ \\
\hline
\end{tabular}

\section{Patient experiences of diagnosis}

Globally, 33\% of respondents $(n=84)$ had been diagnosed with HCC <1 year before completing the survey; $34 \%$ had been living with HCC for $1-3$ years $(n=88)$; and $16 \%(n=42)$ had had HCC for $4-6$ years or longer $(n=42)$ (Table 1). Respondents from The Americas and Asia-Pacific tended to have had HCC for longer than the global average.

Most respondents had concomitant diagnoses: $38 \%$ had hepatitis $\mathrm{C}$ virus $(\mathrm{n}=97), 23 \%$ had hepatitis $\mathrm{B}$ virus $(\mathrm{HBV} ; \mathrm{n}=60)$, while $<10 \%(\mathrm{n}=24)$ had nonalcoholic fatty liver disease (Table 1). Patients from The Americas 
(A)

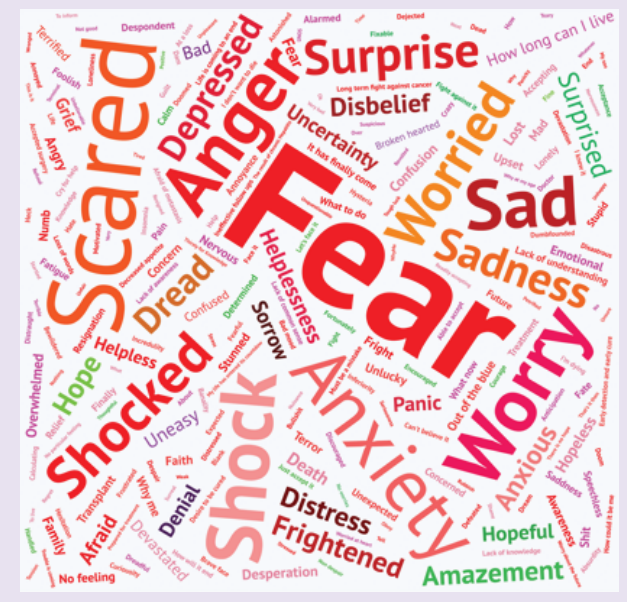

(B)

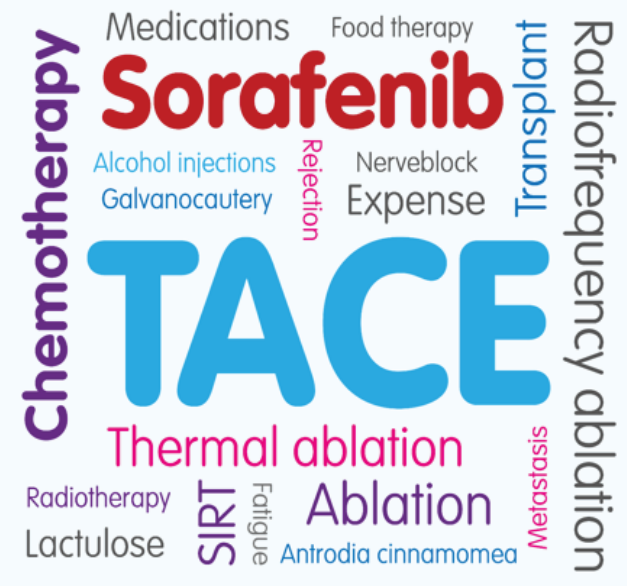

Figure 1. Descriptive word clouds. 'Thoughts on diagnosis' (A) and 'most challenging treatment' (B) word clouds. In both word clouds, the size of the individual word relates to the number of times it was used by respondents. (A) Represents the three words used by respondents to describe their feelings upon diagnosis of HCC. Question: which three words best describe your feelings when you learned you had HCC? (B) Represents the treatment patients reported as being the most challenging (not including surgery). Question: excluding surgery - which of the treatments you have received for your liver cancer has been the most challenging? HCC: Hepatocellular carcinoma.

were least likely to have HBV (4.7\%), while respondents from Asia-Pacific were most likely to have had HBV before their HCC diagnosis (66\%).

Most respondents were diagnosed by either a specialist hepatologist $(43 \%, \mathrm{n}=109)$, or a gastroenterologist $(25 \%, \mathrm{n}=64)$ (Table 1). A higher proportion of patients in the Asia-Pacific $(62 \%, \mathrm{n}=32)$ were diagnosed by a hepatologist than in Europe $(35 \%, \mathrm{n}=32)$ or the Americas $(39 \%, \mathrm{n}=25)$. In the Asia-Pacific $11 \%(\mathrm{n}=7)$ of patients were diagnosed by a gastroenterologist in contrast to $28 \%(n=37)$ in Europe and $32 \%(n=20)$ in the Americas. Globally $15 \%(n=39)$ of patients were diagnosed by their general practitioner, with similar proportions in each region (Table 1$)$.

Respondents were asked for three words that best described their feelings upon diagnosis of HCC. These words are presented as a word cloud in Figure 1A. The five most common words used were 'fear', 'worry', 'scared', 'anxiety' and 'shock', but words like 'hope' and 'calm' were also used by some patients.

Respondents were also asked what sources of information they used at the time of diagnosis, and how useful they found them. The most useful source of information for most respondents was their specialist doctor (Figure 2). Most patients found their specialist to be of good or excellent value in terms of information around HCC (85\%, $\mathrm{n}=218$ ). Friends and family, nurses and primary care doctors were also found to be of good or excellent value for more than half of respondents. Charities or not-for-profit agencies were of limited value, and were not used by $60 \%$ of patients $(n=155)$. Out of the 100 respondents who did use them, $37 \%$ found them to be of limited value $(\mathrm{n}=37)$. Medical websites were used by more than two-thirds of respondents $(\mathrm{n}=178)$, and two-thirds of those using them $(n=113)$ thought they were of good or excellent value.

Patients were asked if they received enough information about HCC and its treatment at the time of their diagnosis. Overall, $68 \%$ of respondents said no $(n=175)$, but this figure varied by region: for example, in Europe $75 \%$ of patients said that they had not received enough information at diagnosis compared with $61 \%$ in the Asia-Pacific region (Table 1).

\section{Impact of treatment}

Patients tended to have undergone more than one treatment during their treatment journey (Table 1); 83\% of respondents had received at least one of the earlier-stage HCC treatments (liver ablation, transplant and surgery combined, $\mathrm{n}=213)$. The most frequently-reported later-stage treatment was TACE $(43 \%, \mathrm{n}=111)$. A similar 


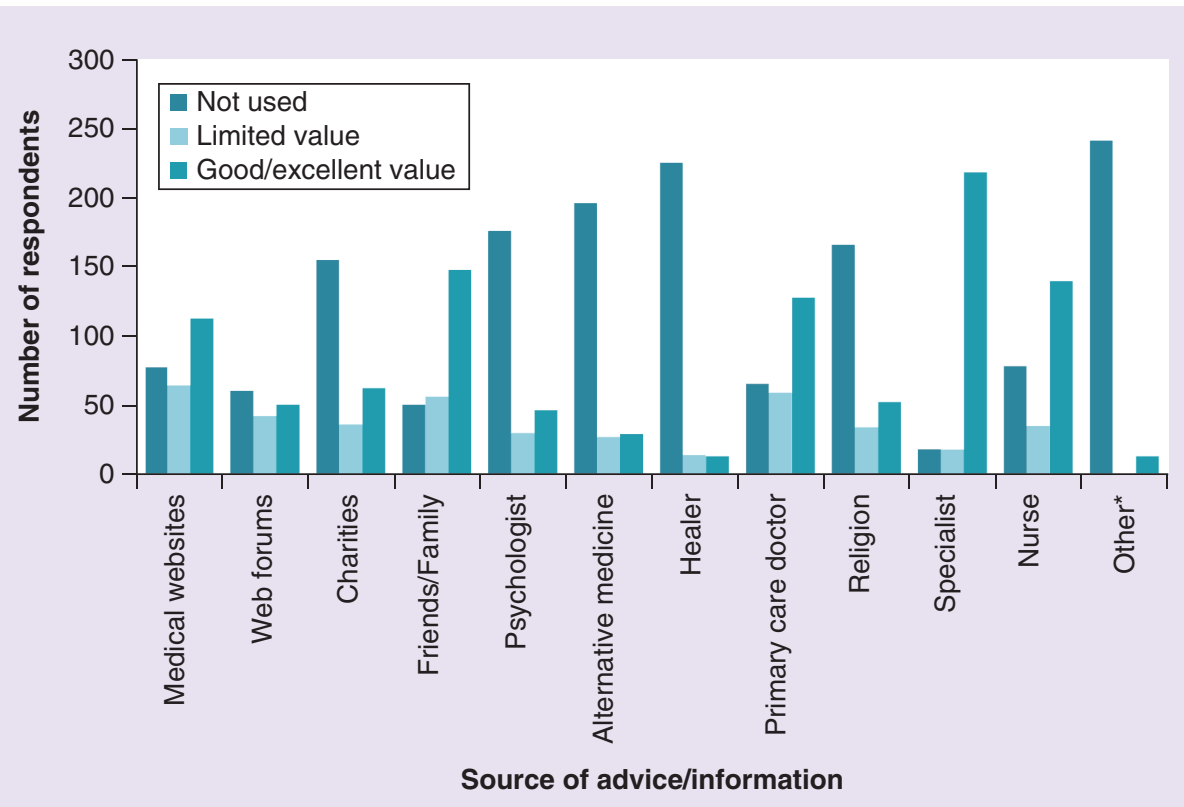

Figure 2. Sources of advice for patients with hepatocellular carcinoma. The number of respondents using different sources of advice/information regarding their HCC diagnosis and treatment, as well as the value of these information sources to those who used them. Question: during your diagnosis or treatment where have you gone for advice or counsel and how useful have you found it? HCC: Hepatocellular carcinoma.

number of patients received external radiotherapy $(4 \%, \mathrm{n}=11)$ and intravenous chemotherapy $(4 \%, \mathrm{n}=10)$. Almost a fifth of respondents were prescribed sorafenib during their treatment $(n=46)$, while $13 \%(n=33)$ were treated with SIRT. A total of $4 \%$ of patients said they had not been offered any treatment, while $2 \%$ declined any treatment offered to them. In Asia-Pacific, surgery (49\%) and ablation (49\%) were more common than in other regions, and transplant was common in The Americas (39\%) (Table 1). Transplant, surgery and ablation were less common in Europe (66\% combined). SIRT was more common in North America (21.9\%) than in Europe (9\%) and Asia-Pacific (13\%).

The patient-reported impact of side effects related to these treatments on the QoL of respondents was analyzed. Abdominal pain was experienced by $60 \%$ of respondents $(n=153)$, skin disorders by $28 \%(n=72)$, nausea by $38 \%(\mathrm{n}=96)$, diarrhea by $30 \%(\mathrm{n}=70)$, fatigue by $63 \%(\mathrm{n}=160)$, weight loss by $37 \%(\mathrm{n}=95)$, alopecia by $18 \%$ $(\mathrm{n}=45)$, and appetite loss by $40 \%(\mathrm{n}=103)$. Out of those experiencing these side effects, skin disorders and fatigue had the biggest impact on QoL; two-thirds of those experiencing any fatigue or skin disorders felt that they had a moderate-to-significant negative impact on their QoL ( $n=103$ and $n=46$, respectively). In contrast, only 44\% of those suffering from alopecia and appetite loss thought it caused a moderate-to-significant reduction in their QoL $(\mathrm{n}=20$ and $\mathrm{n}=45$, respectively).

Out of those who were working at the time they started HCC treatment, $60 \%(\mathrm{n}=154)$ said that the side effects they experienced caused them to stop working. Treatment also negatively affected patients' relationships with their family/carers (28\%), their level of energy (44\%), their ability to perform daily activities (37\%), and their outlook for the future (34\%). Comparing their life before and after their HCC diagnosis, $47 \%$ of patients had a reduced ability to concentrate, $44 \%$ had reduced overall physical condition, and $36 \%$ had a reduced overall mental condition.

Patients were asked to specify which treatment throughout their patient journey they found the most challenging, excluding surgery. Out of those patients that answered this question, 37\% thought that TACE was the most challenging, while a quarter thought that sorafenib was the most challenging (as reflected in the large font for the words 'TACE' and 'sorafenib' shown in word cloud B, Figure 1B).

To determine the specific negative effects of late-stage treatment, respondents were asked what the impact of their most recent treatment had been on their QoL. Answers from people who were given therapies normally used in more 


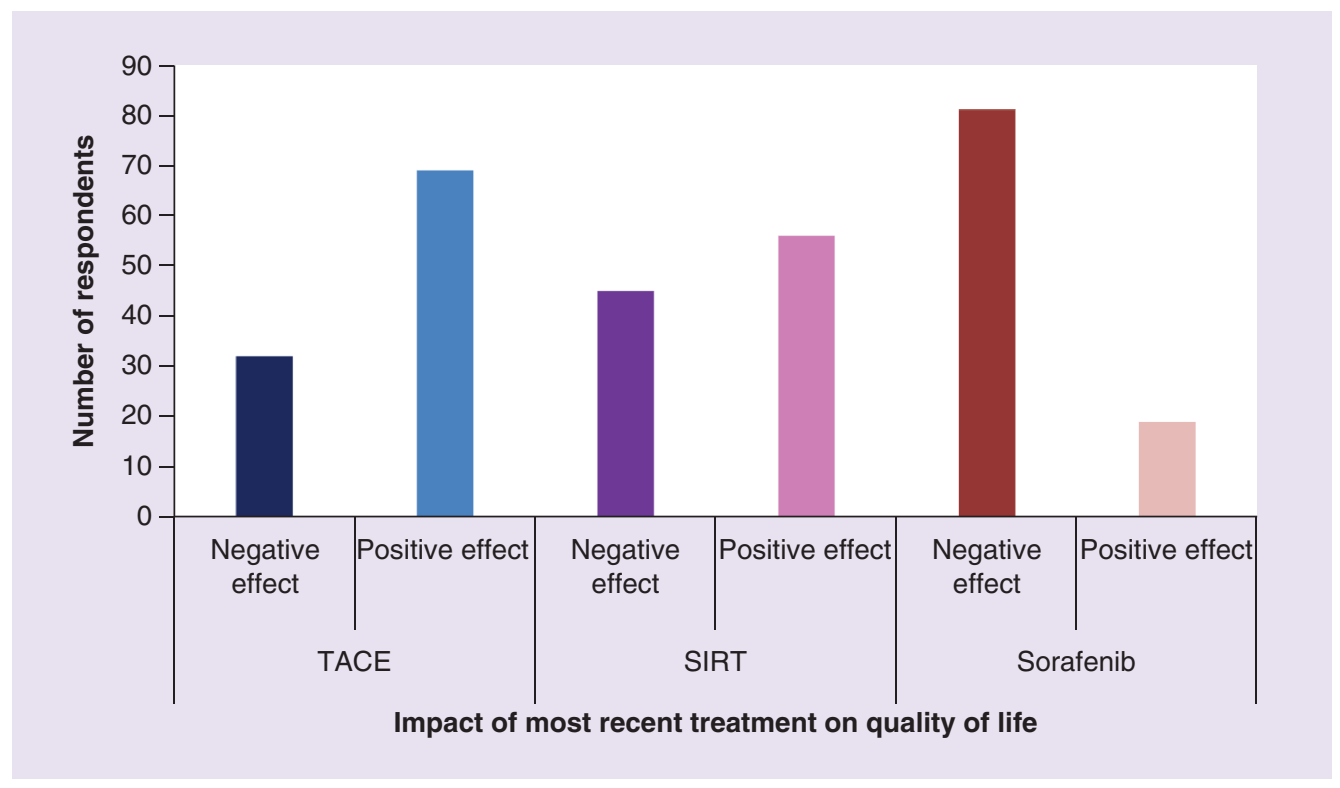

Figure 3. Effect of most recent treatment on quality of life. Comparison of the impact of later stage treatment (TACE, SIRT or sorafenib) on quality of life, which would either have been in a negative or positive direction compared with quality of life before the most recent treatment started. Question: thinking about your present or most recent medical treatment, what has been its effect on your quality of life?

SIRT: Selective internal radiation therapy; TACE: Transarterial chemoembolization.

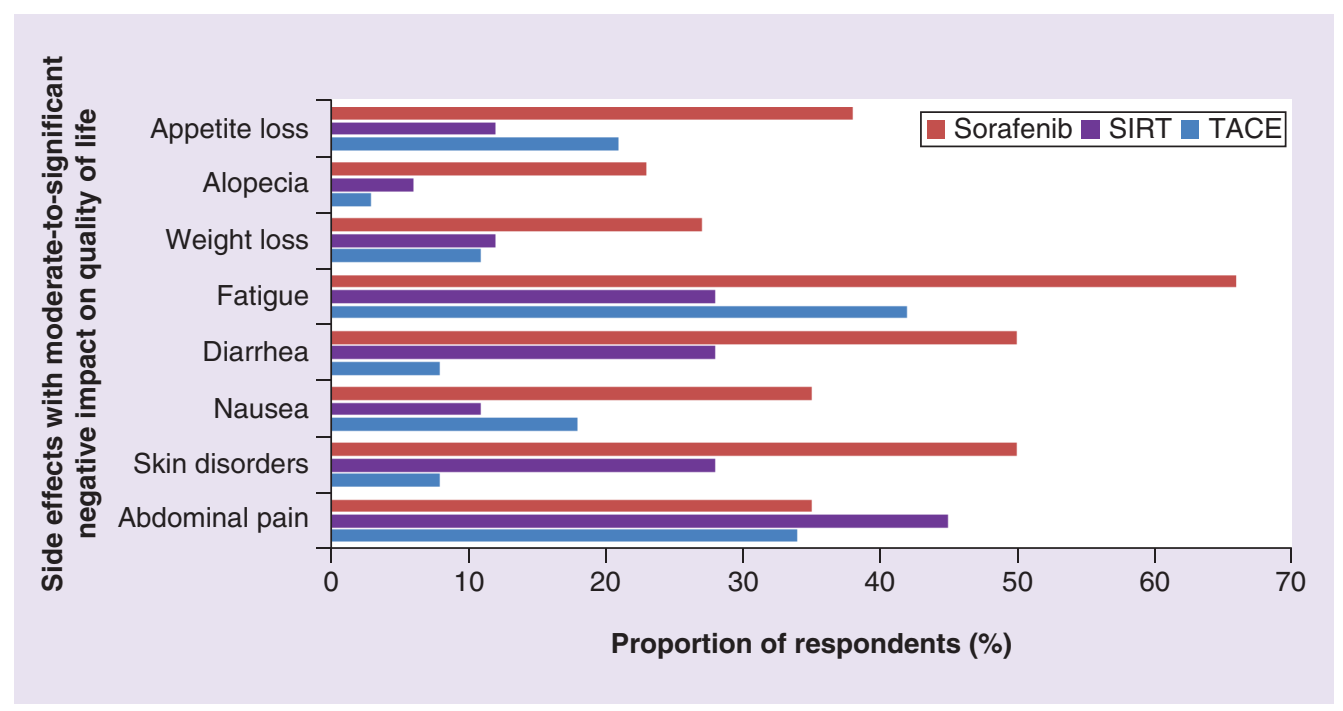

Figure 4. Late stage treatment side effects. Comparison of proportion of respondents whose late stage treatment (TACE, SIRT or sorafenib) related side effects had a 'moderate-to-significant' negative impact on their quality of life. SIRT: Selective internal radiation therapy; TACE: Transarterial chemoembolization.

advanced disease were compared. Most people whose most recent treatment was sorafenib said it had a negative effect on their QoL $(81 \%, n=21)$, while only $19 \%(n=5)$ said their QoL improved due to sorafenib. In contrast, $69 \%(\mathrm{n}=26)$ and $56 \%(\mathrm{n}=10)$ of those using most recently TACE and SIRT, respectively, saw an improvement in their QoL (Figure 3). Similarly, patients taking sorafenib more frequently reported moderate-to-significant side effects affecting QoL. The most common sorafenib-related side effect with a moderate-to-significant impact on QoL was fatigue $(66 \%, \mathrm{n}=17)$, followed by skin disorders $(50 \%, \mathrm{n}=13)$ and diarrhea $(50 \%, \mathrm{n}=13)$ (Figure 4).

Patients were asked to rate their QoL at the time of survey completion (Figure 5). Just over two-fifths of those 


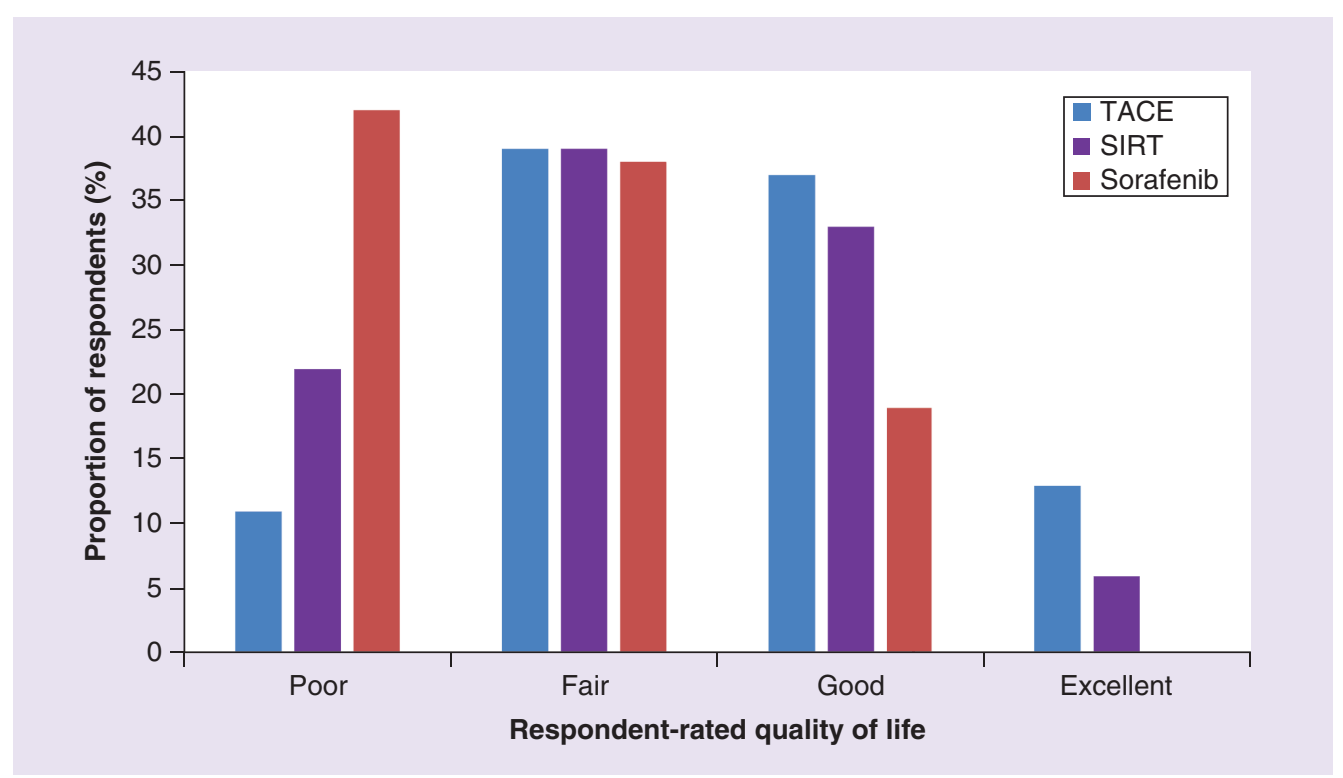

Figure 5. Respondents' rating of their current quality of life. Comparison of respondents' current quality of life rating for those using late-stage treatments (TACE, SIRT or sorafenib). Question: how would you rate your quality of life today?

SIRT: Selective internal radiation therapy; TACE: Transarterial chemoembolization.

taking sorafenib most recently $(42 \%, \mathrm{n}=11)$ reported a poor QoL, while $19 \%(\mathrm{n}=5)$ stated their QoL was good. In contrast, $50 \%(\mathrm{n}=19)$ and 39\% $(\mathrm{n}=7)$ of those treated most recently with TACE and SIRT, respectively, rated their QoL as good or excellent.

\section{Treatment access: supplementary health insurance}

Globally, over a third of respondents reported using supplementary health insurance $(36 \%, n=92)$. This proportion was slightly higher when considering North American respondents in isolation $(48 \%, \mathrm{n}=29)$, but was more than double $(79 \%, \mathrm{n}=48)$ among respondents from the Asia-Pacific region. Out of the $36 \%$ that used supplementary insurance globally, three-quarters said that not all their potential treatment options were covered by their insurance policy. A third of people answering the survey said they had to be referred to an alternative hospital because the treatment option their doctor wanted was not available locally $(36 \%, n=91)$.

\section{Discussion}

To our knowledge, this is the first large-scale, global survey that has attempted to determine the impact of HCC and its treatment modalities from the perspective of the patients. While examples of physician-related surveys [18], surveys of patients with primary liver cancer in specific countries [19], and data around the use of specific treatment modalities exist [20], there is a lack of previously published data on specific experiences of patients with HCC [1-4].

Upon diagnosis of HCC, patients - irrespective of their country - face genuine fear and worry, as is to be expected with the diagnosis of any life-threatening or terminal condition. A cancer diagnosis can impact different aspects of peoples' lives, including psychological, social, and physical dimensions, causing a significant impact on QoL [21]. A diagnosis of cancer is often accompanied by distress, emotional reactions, anxiety, hopelessness, feelings of loneliness and despair. While respondents in this study generally experienced such 'negative' feelings, some reported feelings of 'hope', of being 'determined', feeling 'courage', 'calm', 'positive' or having a need to 'fight' the disease. Nevertheless, the overall feeling upon diagnosis was negative. Patients reported a desire for more information, comfort and support when diagnosed with HCC.

Improving patients' abilities to access useful, understandable information about their condition and the potential treatment options may help to alleviate some of their concerns upon diagnosis. Two-thirds of the patients who responded to this survey did not feel that they received enough information when diagnosed. This is consistent with prior work showing that, upon diagnosis of cancer, patients want significantly more information on the long-term implications of treatment and disease [22], including prognosis and side effects [23]. There is a role globally for more 
specific HCC-related patient advocacy groups and organizations (such as Blue Faery in the USA), which can tailor treatment information specific to the patient journey of those with HCC and ensure information is easily accessible by those who need it. Our results show that patients in Europe reported a higher perceived lack of information than those in The Americas and Asia-Pacific. Perhaps, therefore, there is a specific role for European HCC patient organizations to improve the availability of information.

The most common pre-HCC liver conditions varied across regions, which may impact the specific information requirements of patients in those regions. For example, two-thirds of patients from Asia-Pacific had chronic HBV infection prior to contracting HCC, in contrast to just 5\% of patients from North America. Ensuring effective treatment of HBV in Asia-Pacific, and ensuring patients with HBV in the region are suitably informed of the risks of HCC, may in turn reduce the high incidence of HCC.

Findings from this survey demonstrate the high burden that HCC has on patients' daily lives, including their mood, their energy levels, their ability to exercise and work, their relationships with family and friends, and their overall QoL. The impact of the side effects related to late-stage treatment on QoL indicates the need for additional late-stage treatment options that focus on QoL maintenance.

The survey has some potential limitations that should be considered. First, the nature of the online survey and the absences of a facilitator or interviewer limited the length and complexity of the questionnaire. All findings were also based solely on patient responses, and unconfirmed by medical records; therefore, recall bias may be an issue. Similarly, as most patients were recruited via patient advocacy groups and specialist doctors' offices, or via selfselection following survey-related publications, they may have been more likely to be highly engaged, motivated patients, and thus not fully representative of the overall population of patients with HCC. HCC status, liver reserve status, and factors such as nutritional status, may all impact on the HCC patient's QoL, but gathering this information was beyond the scope of this patient-targeted survey. Although, alcoholic liver disease has an important role in the etiology of HCC, its incidence in the study cohort was not recorded as it was not deemed feasible to collect self-reported information on alcoholism. Despite the availability of hard-copy versions of the survey in some participating clinics, most surveys were completed online (88\%). As such, responses from those patients with HCC without online access may have been limited. Finally, although the survey was developed using an iterative process with HCC experts, it did not incorporate any of the available standardized validated health-related QoL measurement tools [24,25]. Nevertheless, the findings of this survey are consistent with other smaller, single-country studies that reported lower health-related QoL in patients with HCC compared with the general population [26-28].

Notwithstanding the potential limitations of this study, the findings directly reflect patients' perspectives on HCC. This uniquely personal information does help us better understand how patients with HCC directly experience their disease. These valuable insights may in turn focus clinician awareness on the impact of HCC, and encourage the development of new methods to improve patient management and enhance the daily lives of those living with HCC.

\section{Conclusion}

This paper reports the results of a recent global survey of patients living with HCC, and shows that, irrespective of their geographic location, patients with HCC have to confront the genuine fear and anxiety that accompanies the diagnosis of any serious illness.

Most patients responding to the survey felt that they were missing out on much-needed information at diagnosis, suggesting the need for improvement. Patients need more easily accessible information at all stages of their journey, from diagnosis through treatment, to end-of-life care. There is a significant role here for patient advocacy groups and organizations specific to HCC.

Many of the treatments available to patients with HCC had a negative impact on their QoL, but it was the treatment available for patients with more advanced stages of HCC, specifically sorafenib, that tended to elicit the most destructive side effects, and in turn reduce QoL. Given that later-stage treatments have such negative effects, improved treatment strategies, which maintain QoL as far as possible, are required to ensure that patients with advanced, incurable disease do not need to choose between a few months of extra, lower-quality life or a shorter lifespan.

\section{Future perspective}

Future studies of this type could include the physician's perspective, to increase understanding of physician-patient communications. Including the physician's perspective would also enable the influence of other factors on the HCC 
patient's journey to be elucidated. These insights may lead to the development of new methods to improve patient management of HCC and enhance the daily lives of those living with HCC.

\section{Summary points}

- Insights from 256 patients in the first global survey of patients with hepatocellular carcinoma (HCC).

- All patients confronted significant fear and anxiety on diagnosis of HCC.

- Two-thirds of HCC patients do not feel they get enough information about their condition.

- Patients with HCC need more information to better understand their illness.

- Side effects due to current late stage treatments reduce quality of life (QoL).

- Fatigue and pain have the biggest burden on QoL.

- In patients receiving noncurative treatment, those using oral anticancer therapy were more likely to rate their QoL as poor compared with those taking selective internal radiation therapy or TACE.

- Those with incurable HCC need more, and better, treatment options to maintain QoL.

- While survey findings were remarkably consistent across the three regions studied, there were some noteworthy differences, including (as expected) the relative impact of a primary diagnosis with HBV (Asia-Pacific) versus HCV (Europe and the Americas), access to liver transplant (greater in the Americas), and the reliance on supplementary insurance to pay for the costs of treatment (greater in the Americas and particularly so in Asia-Pacific).

\section{Acknowledgements}

The authors would like to thank all patients who participated in this survey and strategic sight for assistance with survey development and data collection.

Financial \& competing interest disclosure

J Gill reports grants and personal fees from Sirtex Medical Limited, UK, during the conduct of the study; grants from Pfizer Inc., outside the submitted work. EM Yoshida reports grants from AbbVie Inc, during the conduct of the study; grants and personal fees from Gilead Sciences, grants and personal fees from Merck Inc, grants from Janssen Inc, grants from Springbank Inc, grants from Intercept Inc, grants from Genfit, personal fees from Celgene Canada Inc, outside the submitted work. This study was funded by an unrestricted educational grant from Sirtex Medical Limited, UK. The authors have no other relevant affiliations or financial involvement with any organization or entity with a financial interest in or financial conflict with the subject matter or materials discussed in the manuscript apart from those disclosed.

No writing assistance was utilized in the production of this manuscript.

\section{Open access}

This work is licensed under the Attribution-NonCommercial-NoDerivatives 4.0 Unported License. To view a copy of this license, visit http://creativecommons.org/licenses/by-nc-nd/4.0/

\section{References}

Papers of special note have been highlighted as: $\bullet$ of interest; $\bullet \bullet$ of considerable interest

1. Balogh J, Victor D 3rd, Asham EH et al. Hepatocellular carcinoma: a review. J. Hepatocell. Carcinoma 3, 41-53 (2016).

2. Ferlay J, Soerjomataram I, Ervik M et al. GLOBOCAN 2012 v1.0, Cancer Incidence and Mortality Worldwide: IARC CancerBase No. 11. 2013).

3. Forner A, Llovet JM, Bruix J. Hepatocellular carcinoma. Lancet 379(9822), 1245-1255 (2012).

4. Mittal S, El-Serag H. Epidemiology of hepatocellular carcinoma: consider the population. J. Clin. Gastroenterol. 47(Suppl.), S2-S6 (2013).

5. Omata M, Cheng AL, Kokudo N et al. Asia-Pacific clinical practice guidelines on the management of hepatocellular carcinoma: a 2017 update. Hepatol. Int. 11(4), 317-370 (2017).

6. Bruix J, Sherman M. American Association for the Study of Liver Diseases. Management of hepatocellular carcinoma: an update. Hepatology 53(3), 1020-1022 (2011).

7. Llovet JM, Bru C, Bruix J. Prognosis of hepatocellular carcinoma: the BCLC staging classification. Semin. Liver Dis. 19(3), 329-338 (1999).

8. Cancer Research UK. Let's beat cancer sooner: survival. www.cancerresearchuk.org/about-cancer/liver-cancer/survival 
9. Forner A, Gilabert M, Bruix J, Raoul JL. Treatment of intermediate-stage hepatocellular carcinoma. Nat. Rev. Clin. Oncol. 11(9), 525-535 (2014).

10. Tsochatzis EA, Fatourou E, O’Beirne J, Meyer T, Burroughs AK. Transarterial chemoembolization and bland embolization for hepatocellular carcinoma. World J. Gastroenterol. 20(12), 3069-3077 (2014).

- Reviews effectiveness of transarterial chemoembolization in the treatment of hepatocellular carcinoma (HCC).

11. Llovet JM, Ricci S, Mazzaferro V et al. Sorafenib in advanced hepatocellular carcinoma. N. Engl. J. Med. 359(4), 378-390 (2008).

-. Key study that demonstrates the efficacy and tolerability of the principal available nonsurgical treatments for HCC.

12. Trojan J, Waidmann O. Role of regorafenib as second-line therapy and landscape of investigational treatment options in advanced hepatocellular carcinoma. J. Hepatocell. Carcinoma 3, 31-36 (2016).

- Key study that demonstrates the efficacy and tolerability of the principal available nonsurgical treatments for HCC.

13. Chow PHW, Gandhi M, Asia-Pacific Hepatocellular Carcinoma Trials Group. Phase III multicentre open-label randomized controlled trial of selective internal radiation therapy (SIRT) versus sorafenib in locally advanced hepatocellular carcinoma: the SIRveNIB study. $J$. Clin. Oncol. 35(Suppl.), A4002 (2017).

-. Key study demonstrating that patients with locally advanced HCC treated with SIRT have better tumor response rates and fewer serious adverse events compared with those treated with sorafenib.

14. Vilgrain V, Pereira H, Assenat E et al. Efficacy and safety of selective internal radiotherapy with yttrium-90 resin microspheres compared with sorafenib in locally advanced and inoperable hepatocellular carcinoma (SARAH): an open-label randomised controlled Phase 3 trial. Lancet Oncol. 18(12), 1624-1636 (2017).

-. Demonstrates that SIRT increased tumor response, reduced the incidence of adverse events and improved quality of life compared with sorafenib, in patients with locally advanced or intermediate stage HCC.

15. Kelley K, Clark B, Brown V, Sitzia J. Good practice in the conduct and reporting of survey research. Int. J. Qual. Health Care 15(3), 261-266 (2003).

16. HCC patient voices survey. http://eprints.lse.ac.uk/84143

17. Word Art. www.wordart.com

18. Kumar A. Current practices in management of hepatocellular carcinoma in India: results of an online survey. J. Clin. Exp. Hepatol. 4(Suppl. 3), S140-S146 (2014).

19. Kudo M, Izumi N, Ichida $\mathrm{T}$ et al. Report of the 19th follow-up survey of primary liver cancer in Japan. Hepatol. Res. 46(5), 372-390 (2016).

20. Soubrane O, Goumard C, Laurent A et al. Laparoscopic resection of hepatocellular carcinoma: a French survey in 351 patients. HPB (Oxford) 16(4), 357-365 (2014).

21. Foley KL, Farmer DF, Petronis VM et al. A qualitative exploration of the cancer experience among long-term survivors: comparisons by cancer type, ethnicity, gender, and age. Psychooncology 15(3), 248-258 (2006).

22. Hawkins NA, Pollack LA, Leadbetter $S$ et al. Informational needs of patients and perceived adequacy of information available before and after treatment of cancer. J. Psychosoc. Oncol. 26(2), 1-16 (2008).

23. Fallowfield L, Ford S, Lewis S. No news is not good news: information preferences of patients with cancer. Psychooncology 4(3), 197-202 (1995).

24. Bowling A. Measuring Health: A Review OfQuality Of Life Measurement Scales. Open University Press, Buckingham, UK (1991).

25. Bowling A. Measuring Disease: A Review OfDisease-Specific Quality OfLife Measurement Scales (2nd Edition). Open University Press, Buckingham, UK (1995).

26. Fan SY, Eiser C, Ho MC. Health-related quality of life in patients with hepatocellular carcinoma: a systematic review. Clin. Gastroenterol. Hepatol. 8(7), 559-564 e551-510 (2010).

27. Kondo Y, Yoshida H, Tateishi R et al. Health-related quality of life of chronic liver disease patients with and without hepatocellular carcinoma. J. Gastroenterol. Hepatol. 22(2), 197-203 (2007).

28. Steel JL, Chopra K, Olek MC, Carr BI. Health-related quality of life: hepatocellular carcinoma, chronic liver disease, and the general population. Qual. Life Res. 16(2), 203-215 (2007). 